\title{
Fangchinoline suppresses conjunctival melanoma by directly binding FUBP2 and inhibiting the homologous recombination pathway
}

Keting Bao ${ }^{1}$, Yongyun $\mathrm{Li}^{2}$, Jinlian Wei ${ }^{1}$, Ruoxi $\mathrm{Li}^{1}$, Jie Yang ${ }^{2}$, Jiahao Shi², Baoli Li', Jin Zhu', Fei Mao (D), Renbing Jia (1D) ${ }^{2}$ and Jian Li (iD)

\begin{abstract}
Conjunctival melanoma (CM) is a rare and fatal ocular tumour with poor prognosis. There is an urgent need of effective therapeutic drugs against CM. Here, we reported the discovery of a novel potential therapeutic target for CM. Through phenotypic screening of our in-house library, fangchinoline was discovered to significantly inhibit the growth of CM cells including CM-AS16, CRMM1, CRMM2 and CM2005.1. Further mechanistic experiments indicated that fangchinoline suppressed the homologous recombination (HR)-directed DNA repair by binding with far upstream element binding protein 2 (FUBP2) and downregulating the expression of HR factors BRCA1 and RAD51. In vitro and in vivo antitumour experiments revealed that fangchinoline increased the efficacy of cisplatin by blocking HR factors and reduced the drug dose and toxicity. In conclusion, our work provides a promising therapeutic strategy for the treatment of CM that is worthy of extensive preclinical investigation.
\end{abstract}

\section{Introduction}

Conjunctival melanoma $(\mathrm{CM})$ is a rare and fatal ocular tumour that accounts for $2 \%$ of all ocular malignancies ${ }^{1}$ and $1.6 \%$ of all non-cutaneous melanomas ${ }^{2}$. CM is more common in the Caucasian population (annual incidence of $0.2-0.5$ per million ${ }^{3}$ ) compared to the Asian population (annual incidence of 0.15 per million ${ }^{4}$ ). It has previously been reported that $\mathrm{CM}$ emerges from the malignant transformation of neuroectoderm-derived melanocytes that migrate to the conjunctiva ${ }^{5}$ and may invade the orbit and eyeball, after which they metastasize to regional and systemic lymph nodes ${ }^{6}$. Currently, the first-line treatment

Correspondence: Fei Mao (maofei@ecust.edu.cn) or

Renbing Jia (renbingjia@sjtu.edu.cn) or Jian Li (jianli@ecust.edu.cn)

${ }^{1}$ State Key Laboratory of Bioreactor Engineering, Shanghai Key Laboratory of New Drug Design, East China University of Science and Technology, 130 Mei Long Road, Shanghai 200237, China

Department of Ophthalmology, Ninth People's Hospital, Shanghai Jiao Tong University School of Medicine, Shanghai 200001, China

Full list of author information is available at the end of the article

These authors contributed equally: Keting Bao, Yongyun Li, Jinlian Wei

Edited by S. Inoue for CM is wide local excision and biopsy with 'no touch' technique ${ }^{7}$, followed by adjunct treatment with cryotherapy, radiotherapy ${ }^{8}$ and chemotherapy using mitomycin $C$ or interferon $\alpha-2 b^{9}$. Among the Caucasian CM patients in a Dutch referral centre, the overall 5-year recurrence rate, metastasis rate and melanoma-related survival rate were reported to be $29 \%, 12 \%$ and $90 \%$, respectively ${ }^{10}$. In another study on Chinese CM patients, the 10-year tumour-related recurrence rate, metastasis rate and mortality were observed to be $66 \%, 51 \%$ and $37 \%$, respectively. The results of a large retrospective study on CM indicated that the Chinese CM patients presented a more compromised prognosis compared to the Caucasian CM patients, which might be attributed to the limited standard medical treatment available in rural areas, as well as the prevalence of varied tumour cell types among different races ${ }^{11}$.

Mutations in BRAF and NRAS are commonly observed in cutaneous melanoma, with incidences of $37 \%-50 \%$ and $13 \%-25 \%$, respectively ${ }^{12}$. A previous study on 78 Caucasian cases reported that $29 \%$ of CM patients harboured

\section{(c) The Author(s) 2021}

(c) (i) Open Access This article is licensed under a Creative Commons Attribution 4.0 International License, which permits use, sharing, adaptation, distribution and reproduction cc) in any medium or format, as long as you give appropriate credit to the original author(s) and the source, provide a link to the Creative Commons license, and indicate if changes were made. The images or other third party material in this article are included in the article's Creative Commons license, unless indicated otherwise in a credit line to the material. If material is not included in the article's Creative Commons license and your intended use is not permitted by statutory regulation or exceeds the permitted use, you will need to obtain permission directly from the copyright holder. To view a copy of this license, visit http://creativecommons.org/licenses/by/4.0/. 
$B R A F$ mutation, whereas $18 \%$ harboured NRAS mutation $^{13}$. However, another study on Chinese CM population revealed that $B R A F$ mutation was detected in only $8 \%$ of the patients ${ }^{14}$, indicating that different races had different genetic mutation profiles. Notably, Caucasian patients with NRAS-mutant melanoma presented a more aggressive natural history and poorer survival rates than those with $B R A F$-mutant or wild-type melanoma ${ }^{15,16}$. To date, the mitogen-activated protein kinase (MEK) inhibitor, MEK162, is the only Food and Drug Administration-approved targeted drug used for the treatment of advanced cutaneous melanoma with NRAS mutation $^{17}$. No BRAF or NRAS mutation-targeting drugs in CM patients have been approved, although some specific inhibitors have been shown to exhibit positive effects in clinical trials ${ }^{18,19}$. This has given rise to the need for developing novel therapeutic targets for CM patients.

Due to the unmet need, a phenotypic screening of our in-house 1347 marketed drugs against CM was conducted in CM-AS16 cells obtained from a Han Chinese patient with a typical NRAS mutation ${ }^{20}$ for potential candidate drugs. Our group focuses on drug repurposing and has achieved some success ${ }^{21,22}$. After an initial screening, cepharanthine was discovered to inhibit the proliferation of CM-AS16 cells. Meanwhile, its analogue fangchinoline showed comparable activity and was selected for further study. Fangchinoline inhibits the growth of various tumours ${ }^{23}$, but its anti-CM effect remains unknown. The transcriptome analysis demonstrated that fangchinoline suppressed the homologous recombination (HR) pathway in CM-AS16 cells. Furthermore, an activity-based protein profiling (ABPP) chemoproteomic approach was used to map the proteome-wide targets of fangchinoline in CMAS16 cell proteome. It revealed FUBP2 to be one of the primary targets of fangchinoline. Furthermore, fangchinoline inhibited the expressions of downstream proteins of FUBP2, including c-Myc ${ }^{24}$, BRCA1 and RAD51 ${ }^{25}$. All of these results may contribute to the development of novel CM-targeted therapies.

\section{Results}

Fangchinoline inhibited the growth of CM cells in vitro and in vivo

The anti-proliferative activity of our in-house drugs against CM-AS16 cells was assessed using the Cell Counting Kit-8 (CCK-8) assay at a final concentration of $10 \mu \mathrm{M}$ (Fig. 1A). Of them, 41 drugs exhibited good inhibitory activity (inhibition rate $>50 \%$ ) and were selected for further $\mathrm{IC}_{50}$ investigation in both CM-AS16 and another CM cell line CRMM2. Our results indicated that an approved natural drug cepharanthine significantly inhibited the growth of CM-AS16 cells with $\mathrm{IC}_{50}$ value of $1.63 \mu \mathrm{M}$. The other three cepharanthine analogues, berbamine hydrochloride, tetrandrine and fangchinoline, also inhibited CM-AS16 cell growth with $\mathrm{IC}_{50}$ values of 8.46, 3.20 and $5.67 \mu \mathrm{M}$, respectively (Supplementary Table S1). These analogues were Chinese herbal monomers harbouring the same bisbenzylisoquinoline core. Among them, fangchinoline exhibited a favourable structural plasticity (Fig. 1A, B). Fangchinoline harbours an exposed hydroxy group, which is suitable for synthesizing functionalized probes and subsequent target identification. Hence, it was selected for an in-depth investigation. Next, we assessed the anti-proliferative activity of fangchinoline against three other CM cell lines obtained from Caucasian patients with a typical NRAS (CRMM2) or BRAF mutation (CRMM1 and CM2005.1) 26,27. Consistent with its potent antitumour activity against CM-AS16 cells, fangchinoline inhibited the cell proliferation in CRMM1, CRMM2 and CM2005.1 cells, with $\mathrm{IC}_{50}$ values of 2.68, 3.60 and $7.40 \mu \mathrm{M}$, respectively (Supplementary Table S1). To determine whether fangchinoline could suppress CM properties in vivo, an immunodeficient NCG mice xenograft model was created by subcutaneous inoculation of CM-AS16 cells. NCG mice comes from knocking out the Prkdc and IL2rg genes in NOD/Shiltt mice through Crispr Cas9 technology. As shown in Fig. 1C, the CM-AS16 tumour growth was significantly inhibited after intraperitoneal (i.p.) treatment of the NCG male mice with fangchinoline at a dosage of $50 \mathrm{mg} / \mathrm{kg} /$ day compared to the control group $(p<0.01)$. The average tumour volume of the control group was $204 \mathrm{~mm}^{3}$, which was higher than that of the treated groups $(50 \mathrm{mg} / \mathrm{kg} /$ day fangchinoline, tumour volume: $149 \mathrm{~mm}^{3}$ and $25 \mathrm{mg} / \mathrm{kg} /$ day fangchinoline, tumour volume: $200 \mathrm{~mm}^{3}$ ). The average tumour volume of group treated with $50 \mathrm{mg} / \mathrm{kg} /$ day fangchinoline decreased by about $27 \%$ compared to the control group, without any significant change in the average body weight (Fig. 1D). Similarly, there were no differences in the levels of alanine transaminase (ALT), aspartate transaminase (AST), creatinine or urea in mice serum of the fangchinoline-treated and control groups, indicating that fangchinoline does not have an impact on liver and renal functions (Supplementary Fig. S1).

\section{Fangchinoline suppressed the HR pathway in CM-AS16 cells}

To unravel the anti-proliferation mechanism of fangchinoline in CM-AS16 cells, transcriptome analysis was performed. As fangchinoline inhibited the cell proliferation of CM-AS16 cells with $\mathrm{IC}_{50}$ values within $5-8 \mu \mathrm{M}$, the concentration of $8 \mu \mathrm{M}$ was selected for transcriptome analysis. To identify the differentially expressed genes (DEGs) between fangchinoline and control groups, the expression of each transcript was calculated according to the fragments per kilobase of exon per million mapped reads method. The Kyoto encyclopedia of genes and genomes (KEGG) enrichment was performed via Fisher's 


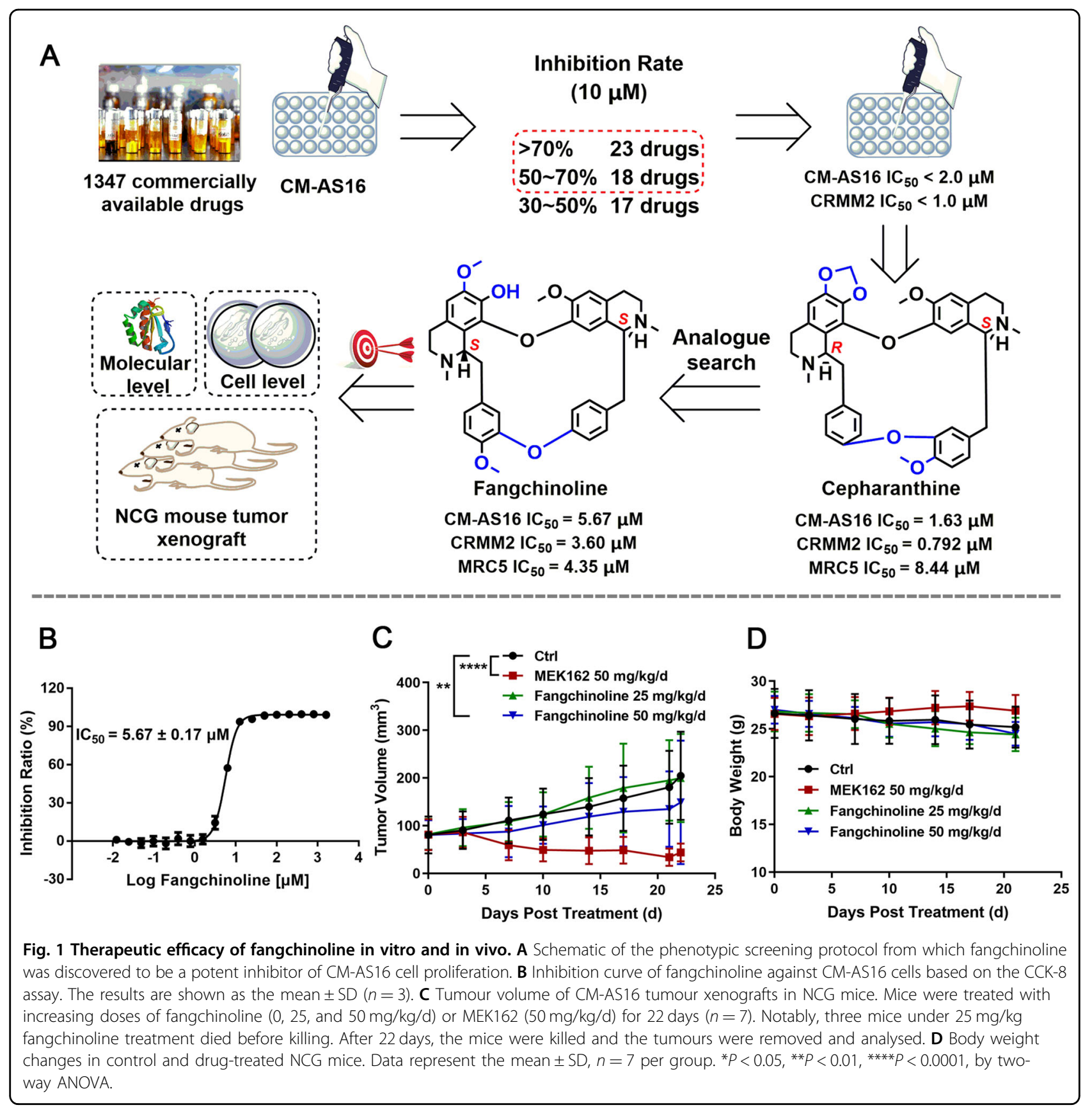

exact test using KOBAS software and was performed to identify which DEGs were enriched. It revealed that nine signalling pathways were significantly affected by fangchinoline treatment (adjusted $P<0.01$ ) and the topranking functional cluster was the HR pathway, with an adjusted $P$-value $<1 \times 10^{-5}$ (Fig. $2 \mathrm{~A}$ ). HR is a critical cellular process that contributes to DNA repair and DNA damage response (DDR). When exposed to genotoxic stresses, cells use the HR pathway to repair DNA doublestranded breaks (DSBs) and interstrand crosslinks (ICLs) with high fidelity ${ }^{28}$. HR is generally mediated by a repertoire of conserved factors, including the essential recombinase, RAD51. Cells deficient in RAD51 are unviable due to its indispensable role in the $H R$ pathway ${ }^{29}$.

Transcriptome analysis of fangchinoline-treated cells suggested significant changes at the transcriptional level in genes associated with the DNA repair and DDR pathways, including the HR pathway (Fig. 2B). Subsequently, we used quantitative real-time PCR (qRT-PCR) to detect the expression of some important genes involved in the HR pathway: BRCA1, BRCA2, RAD50 and RAD51. In line 

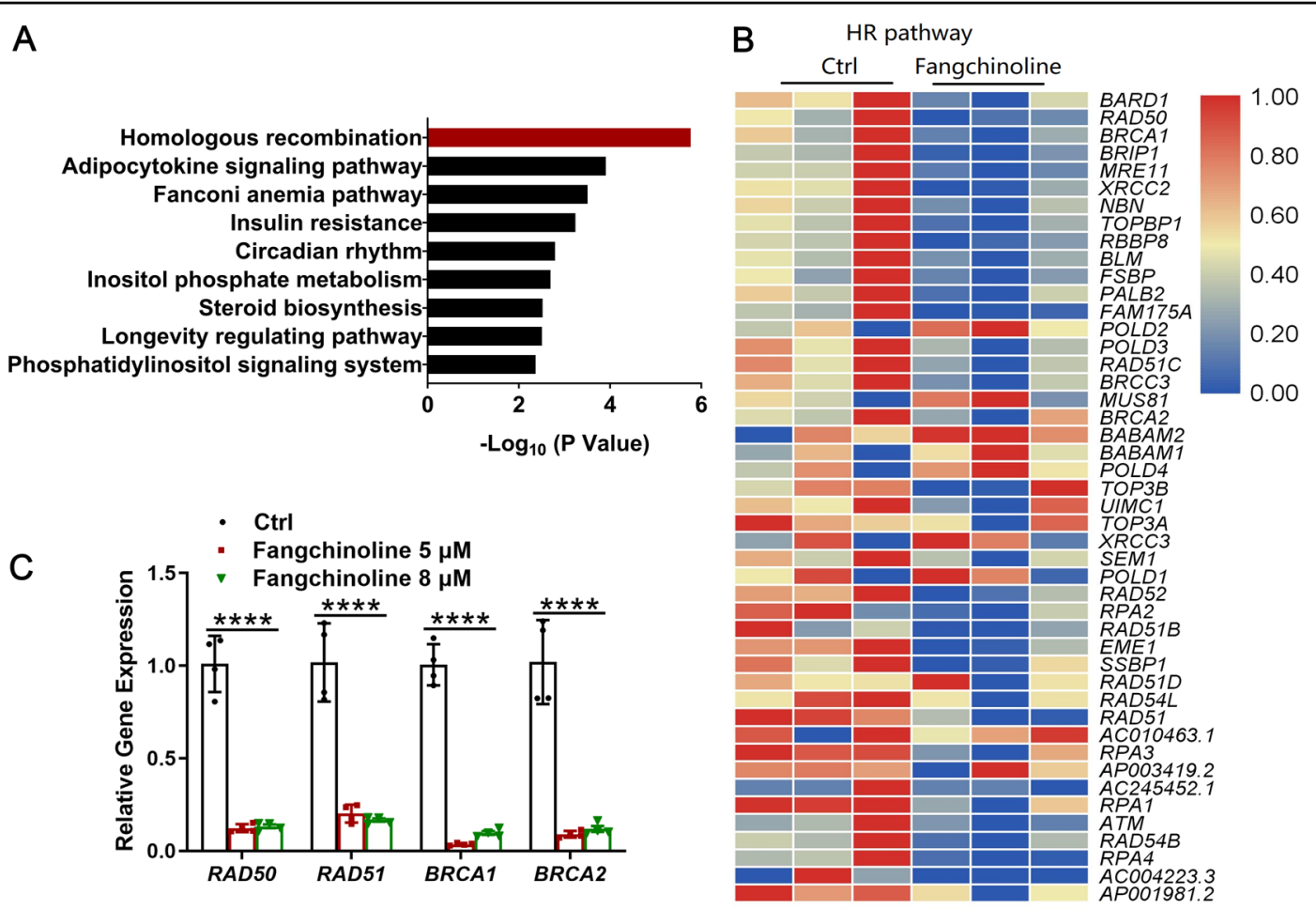

Fig. 2 The effects of fangchinoline on the HR pathway in CM-AS16 cells were determined by transcriptome analysis and qRT-PCR. A KEGG enrichment analysis of DEGs altered by $8 \mu \mathrm{M}$ fangchinoline. All pathways significantly enriched in the DEGs (adjusted $P<0.01$ ) were included. $\mathbf{B}$ A heat map depicting DEGs altered by fangchinoline revealed a significant correlation with the HR pathway. The colour indicates the relative gene expression value. Blue: lowest expression; red: highest expression. C Quantification of the relative expression of genes related to the HR pathway. Data represent the mean \pm SD of four independent experiments. ${ }^{*} P<0.05,{ }^{* * *} P<0.0001$, by Student's $t$-test.

with these results, we observed that these genes were significantly downregulated in CM-AS16 cells treated with 5 and $8 \mu \mathrm{M}$ fangchinoline (Fig. 2C). Taken together, fangchinoline was responsible for HR suppression in CMAS16 cells.

\section{Fangchinoline targets FUBP2 in CM-AS16 cells}

Although fangchinoline exhibited anti-proliferative properties against many types of cancer cells ${ }^{23}$ including CM cells (as shown in this study), its direct targets are still undefined. To explore the mechanisms of fangchinoline, ABPP assay was conducted. ABPP is one of the chemical proteomic approaches that use smallmolecule chemical probes to elucidate the interactions between compounds and targets, which is widely used for the discovery of functional targets of bioactive compounds $^{30}$. First, we designed and synthesized a photoaffinity fangchinoline probe that contained a diazirine photo-cross-linking group and a 'clickable' handle consisting of an alkynyl group (Fig. 3A and Supplementary Chemical Synthesis). This fangchinoline probe retained its original anti-proliferative activity against CM-AS16, CRMM1, CRMM2 and CM2005.1 cells, with $\mathrm{IC}_{50}$ values of $0.95,1.90,4.26$ and $3.68 \mu \mathrm{M}$, respectively
(Supplementary Table S1). The negative probe had no effect on the growth of CM-AS16 cells with an inhibition rate below $10 \%$ under the concentration of $5 \mu \mathrm{M}$ (Fig. 3B). The overall scheme of the ABPP experiments is shown in Fig. 3C. Briefly, the probes were separately incubated in CM-AS16 cell lysates, followed by ultraviolet (UV)-induced photo-cross-linking. Next, the probe-labelled mixture was conjugated with azide-biotin via click chemistry and enriched on streptavidin columns. Finally, nine proteins were collectively identified as potential targets that specifically bound to fangchinoline (Supplementary Table S2). As fangchinoline effectively blocked the HR pathway, FUBP2 was selected because of its crucial role in DNA repair and DDR. FUBP2 is a multifunctional nucleic acid-binding protein belonging to the FUBP family. It plays an important role in cancer invasion and has been identified as a metastasis-associated candidate molecule. In addition, this protein controls the transcription of protooncogenes such as $c-M y c^{31}$ (which is overexpressed in more than half of human cancers) and is associated with tumour initiation, progression and maintenance ${ }^{32}$. As a transcription factor, c-Myc directly upregulates the HR factors, BRCA1 and RAD51 $1^{33,34}$. 


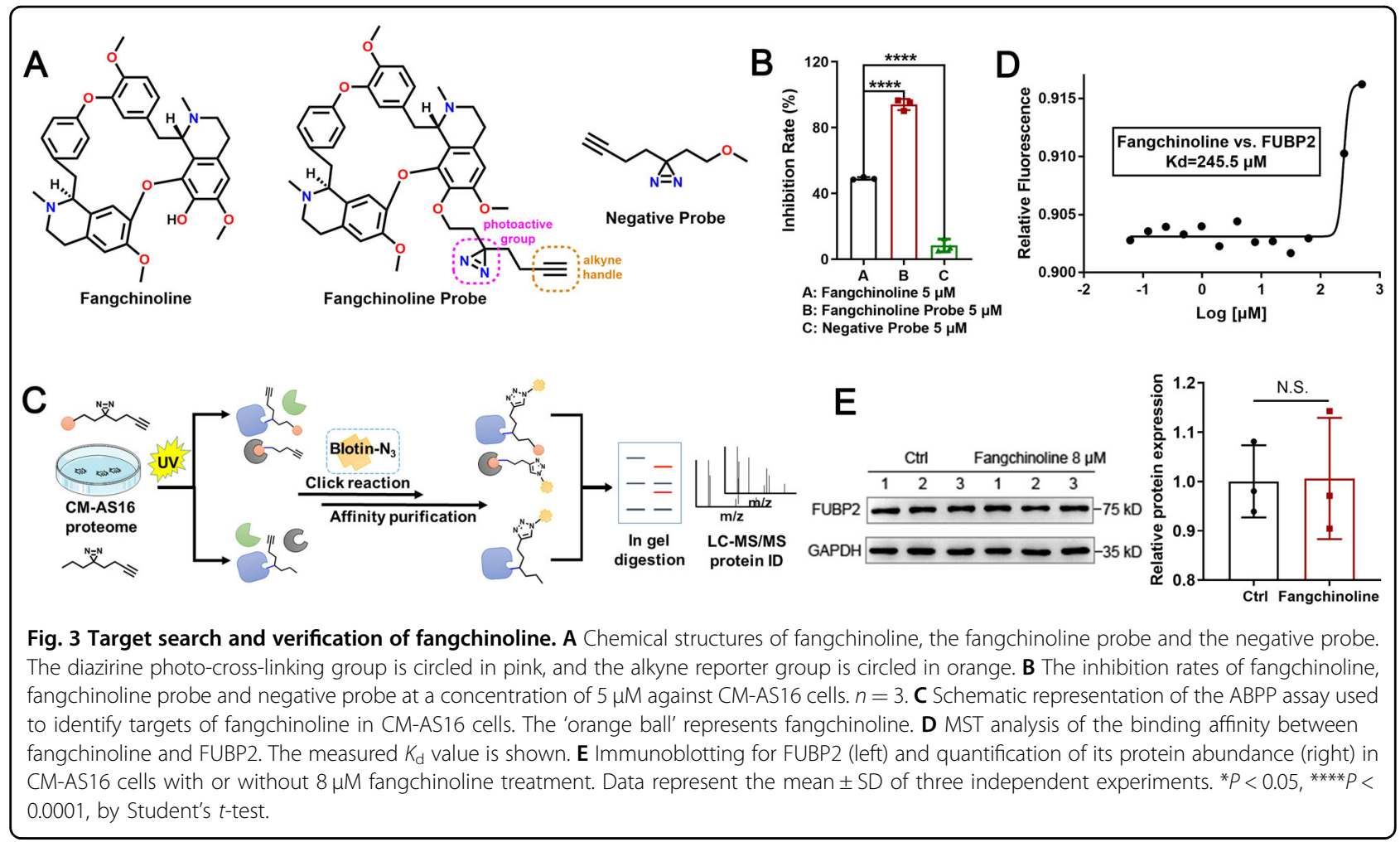

A microscale thermophoresis (MST) assay showed that fangchinoline directly bound to FUBP2 with an equilibrium dissociation constant $\left(K_{\mathrm{d}}\right)$ of $245.5 \mu \mathrm{M}$ (Fig. 3D). In addition, the immunoblotting assays showed that treatment with $8 \mu \mathrm{M}$ fangchinoline did not impact the FUBP2 protein level in CM-AS16 cells (Fig. 3E). These data demonstrated that fangchinoline bound to FUBP2 without affecting its expression.

\section{Fangchinoline suppressed the HR pathway and increased the sensitivity of CM-AS16 cells to DNA damage-inducing drugs}

As FUBP2 upregulated $c-M y c$ transcriptional level, we first evaluated the expression of $c-M y c$ after fangchinoline treatment in CM-AS16 cells. As shown in Fig. 4A, the mRNA level of $c-M y c$ sharply declined after treatment with $8 \mu \mathrm{M}$ fangchinoline in CM-AS16 cells. Meanwhile, a significant dose-dependent decrease in c-Myc protein level was observed in fangchinoline-treated CM-AS16 cells (Fig. 4B). Furthermore, as expected, immunoblotting and immunofluorescent assays of the CM-AS16 grafts also showed a decrease in c-Myc level after administration of $50 \mathrm{mg} / \mathrm{kg} /$ day fangchinoline (Fig. $4 \mathrm{C}$, D). As BRCA1 and $R A D 51$ are transcriptionally regulated by $\mathrm{c}-\mathrm{Myc}$, we evaluated the effect of fangchinoline on their expression in CM-AS16 cells. Consistent with the results from previous qRT-PCR analysis, BRCA1 and RAD51 expressions were inhibited at both transcriptional and protein levels
(Figs. 2C and 4E). Meanwhile, the CM-AS16 grafts also showed that the levels of BRCA1 and RAD51 decreased after administration of $50 \mathrm{mg} / \mathrm{kg} /$ day fangchinoline (Fig. 4F).

Platinum-based agents have been widely used to treat cancers by blocking DNA replication and transcription, as they can lead to generation of various types of DNA adducts, such as ICLs ${ }^{35,36}$. However, the cells have certain repair mechanisms to resolve these lesions. The HR pathway allows the cells to cope with genotoxic stresses by repairing DNA DSBs and ICLs, which explains why BRCA1/2-deficient cancer cells are hypersensitive to platinum compounds ${ }^{28,37}$. BRCA1 and RAD51 play a central role in HR-mediated DSB repair ${ }^{38,39}$ and mediate the response of tumour cells to cisplatin ${ }^{40,41}$. As fangchinoline substantially decreased BRCA1 and RAD51 levels, we further assessed whether combined administration of fangchinoline and platinum compounds could be used as a safe and effective therapy.

As the formation of RAD51 foci is regarded as a critical step in HR, we determined whether the formation of these foci is impaired by fangchinoline ${ }^{42}$. As shown in Fig. 4G, treatment with fangchinoline for $24 \mathrm{~h}$ significantly attenuated the number of RAD51 foci in CM-AS16 cells, which were induced by treatment with cisplatin for $24 \mathrm{~h}$. This result further supported our finding that fangchinoline functionally suppressed the HR pathway in CM cells. 


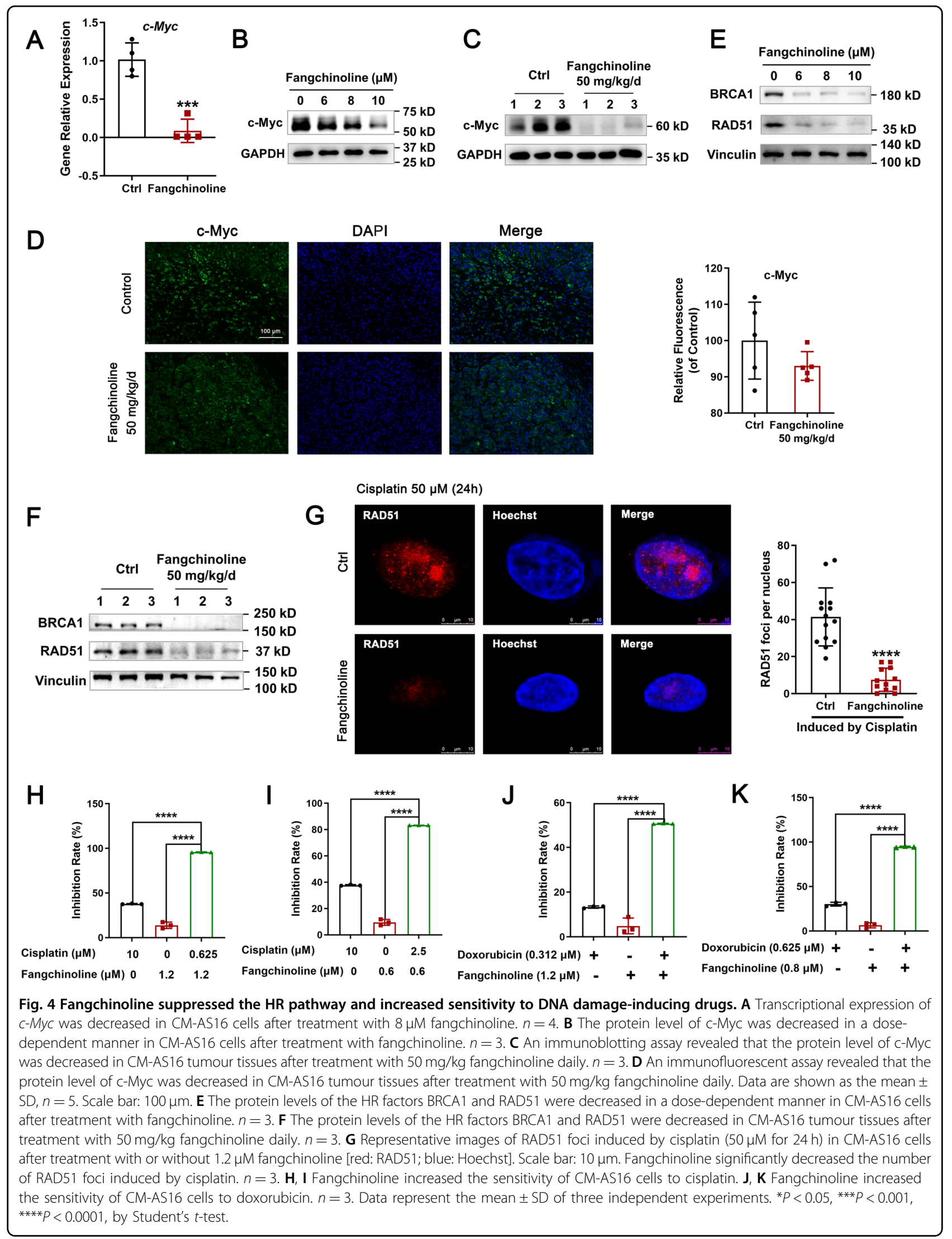


To further confirm these results, we assessed the combined effect of fangchinoline and cisplatin in CM-AS16 cells. CM-AS16 cells were treated with different concentrations of cisplatin in the presence of $0.6 \mu \mathrm{M}$ or $1.2 \mu \mathrm{M}$ fangchinoline. As shown in Fig. $4 \mathrm{H}$, I, both cisplatin $(10 \mu \mathrm{M})$ and fangchinoline $(0.6$ or $1.2 \mu \mathrm{M})$ showed $<50 \%$ inhibition when applied alone. However, combination of these two drugs lead to a significant synergistic effect. In addition, the combination of fangchinoline with another DNA damage-inducing drug doxorubicin also led to a significant synergistic effect (Fig. 4J, K), which indicated that fangchinoline treatment increased the sensitivity of CM-AS16 cells to cisplatin and doxorubicin by interfering with DNA repair and promoting DNA damage.

We next examined the in vivo antitumour effects of fangchinoline, cisplatin and their combination. As shown in Supplementary Fig S9A, in A375 cells, fangchinoline downregulated the expressions of $\mathrm{c}-\mathrm{Myc}, \mathrm{BRCA} 1$ and RAD51. Meanwhile, in consideration of the limited growth rate of CM-AS16 cells, we chose A375 cutaneous melanoma cells for further research. As shown in
Supplementary Fig. S2a, the potency of a combination of fangchinoline and cisplatin was significantly greater than that of fangchinoline or cisplatin alone. Taken together, our results suggested that fangchinoline increased the in vivo antitumour efficacy of cisplatin and reduced its dosage and toxicity. Moreover, we observed that fangchinoline downregulated the expression of c-Myc in the A375 tumour tissues (Supplementary Fig. S3), which indicated that fangchinoline and cisplatin acted synergistically to induce a higher degree of DNA damage. Furthermore, combined fangchinoline and cisplatin treatment increased the proportion of terminal deoxynucleotidyl transferase-mediated dUTP biotin nick end labelling (Tunel)-positive cells (Supplementary Fig. S2b) compared to tissues treated with either fangchinoline or cisplatin, which indicated that fangchinoline conferred increased sensitivity to cisplatin.

\section{FUBP2 facilitated the HR pathway}

To investigate the role of FUBP2 in HR pathway, genetic silencing experiments were conducted. As shown in Fig. 5A,
A

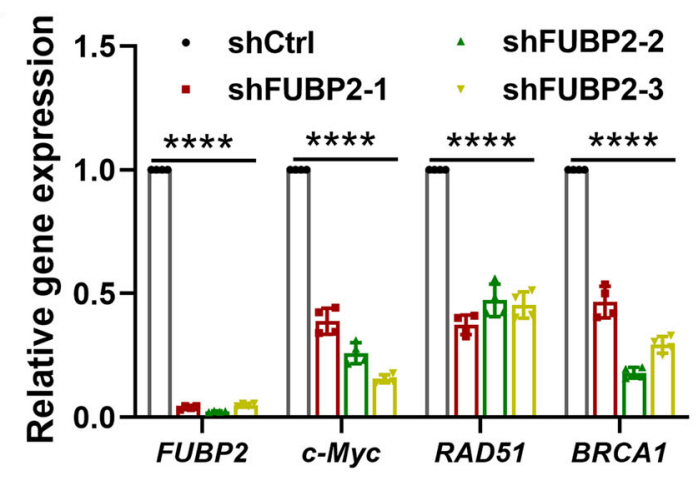

B

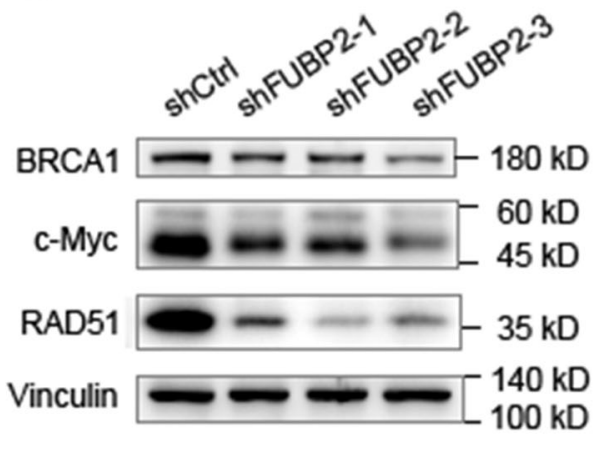

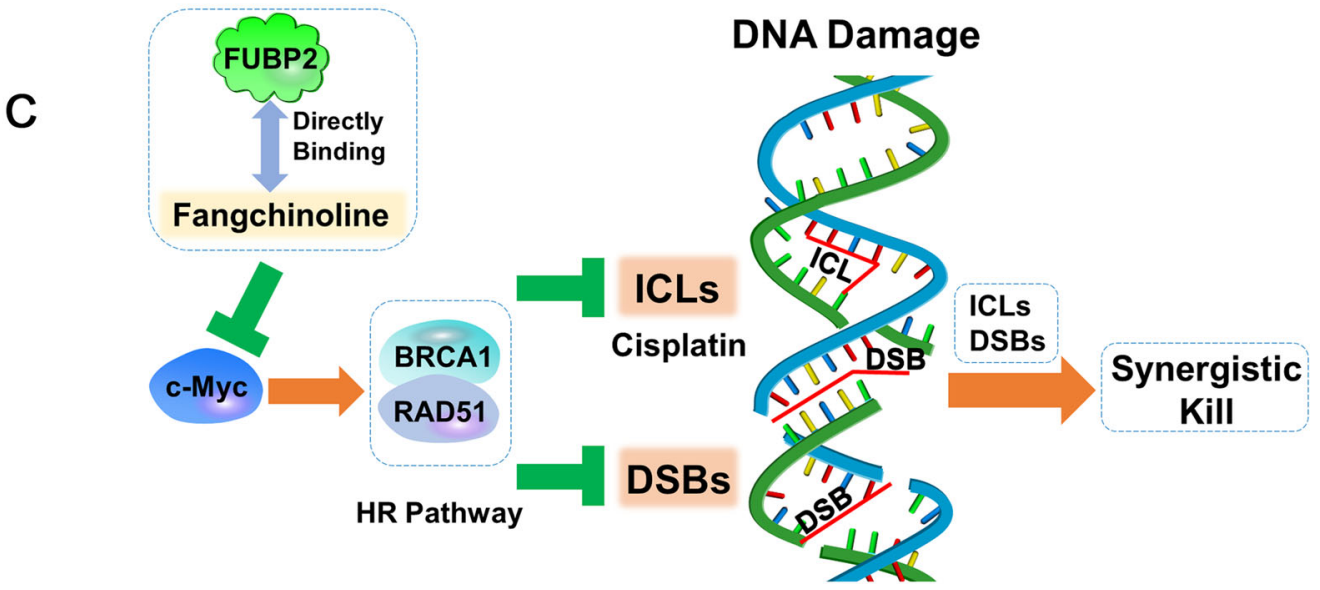

Fig. 5 Effects of FUBP2 on the HR pathway. A The transcriptional expressions of FUBP2, C-Myc, RAD51 and BRCA1 were reduced after treatment with shFUBP2s in CM-AS16 cells. $n=4$. B The protein expressions of c-Myc, RAD51 and BRCA1 were decreased in CM-AS16 cells after treatment with shFUBP2s. $n=3$. C Schematic diagram of the action of fangchinoline in regulating the HR pathway. Data represent the mean \pm SD of at least three independent experiments. ${ }^{*} P<0.05,{ }^{* * * *} P<0.0001$, by two-way ANOVA. 
B, FUBP2 knockdown significantly decreased the expression of c-Myc and HR factors (BRCA1 and RAD51) at both the mRNA and protein level in CM-AS16 cells, which suggested that FUBP2 silencing suppressed HR-directed DNA repair and lead to DNA damage. Overall, the results indicated that fangchinoline significantly inhibited the HR pathway by binding to FUBP2 and downregulating the expressions of c-Myc, BRCA1, and RAD51. Thus, fangchinoline could potentially increase the cellular sensitivity to DNA damage-inducing drugs and induce synergistic killing effect (Fig. 5C).

Fangchinoline suppressed the HR pathway and increased the sensitivity of other CM cells to DNA damage-inducing drugs

To explore whether the effects of fangchinoline treatment in CM-AS16 cells is applicable in the other CM cells too, CM2005.1, CRMM1 and CRMM2 cells were treated with fangchinoline or shFUBP2. As shown in Supplementary Figs. S6A-C, G, H, S7A-C, G, H and S8A-C, G, $\mathrm{H}$, fangchinoline or shFUBP2 treatment in the other three CM cells reduced the expressions of c-Myc, RAD51 and BRCA1 at both the mRNA and protein levels by binding to FUBP2. Moreover, as shown in Supplementary Figs. S6D-F, S7D-F and S8D-F, fangchinoline significantly reduced the formation of cisplatin-induced RAD51 foci and increased the sensitivity to cisplatin and doxorubicin in the other three CM cells too.

\section{Discussion}

In recent years, several signalling pathways, such as the FAK, MEK-ERK1/2, NF- $k B$, and PI3K-Akt-mTOR, have been proposed as the targets of fangchinoline owing to its anticancer activity, which suggested the broad activity spectrum of this herbal monomer in different tumour cells $^{23}$. Herein, our results showed that fangchinoline inhibited the proliferation of CM-AS16 cells both in vitro and in vivo. Notably, this compound reduced the expression of c-Myc at both the mRNA and protein levels by binding to FUBP2, which is also a potential therapeutic target for anti-melanoma ${ }^{43}$. Furthermore, fangchinoline downregulated the expressions of BRCA1 and RAD51 in CM-AS16 cells, which were the downstream effectors of $\mathrm{c}-\mathrm{Myc}$ in the HR pathway. In addition, fangchinoline inhibited the growth of other types of CM cells, including CM2005.1, CRMM1 and CRMM2 cells, through the same mechanism (Supplementary Figs. S6A-C, S7A-C and S8A-C). Moreover, the efficacy of Fangchinoline-FUBP2c-Myc-HR axis was not only observed in CM cells but has also been found to be crucial in other types of cancer cells such as A375, K562, A549, ISK and SKOV3 (Supplementary Fig. S9).

Cisplatin-resistant cells exhibit an elevated DNA-repair ability $^{44}$, which, in turn, increases the survival of cancer cells. Therefore, the development of small molecules that modulate or disrupt the HR pathway has recently attracted increasing attention ${ }^{45,46}$. In our study, we observed that fangchinoline significantly reduced the formation of cisplatin-induced RAD51 foci and inhibited the proliferation of CM-AS16 cells by impairing DNA repair. Thus, fangchinoline could potentially be used as a sensitizing agent in chemotherapy. Similar effects have been observed for other types of $\mathrm{CM}$ cells, including CM2005.1, CRMM1 and CRMM2 cells (Supplementary Figs. S6D-F, S7D-F and S8D-F). As expected, fangchinoline significantly downregulated the levels of c-Myc, BRCA1 and RAD51, both in vitro and in vivo, increased the sensitivity to cisplatin and decreased the drug dosage and toxicity, which could be used to overcome cisplatin resistance.

In conclusion, our study revealed a novel and unique mechanism of fangchinoline that leads to the suppression of CM. Fangchinoline can be used as an adjuvant drug in the therapy of CM or other kinds of cancer and exhibits great potential as a candidate drug in combination therapies.

\section{Materials and methods \\ Reagents}

Fangchinoline (98\%) was from Chengdu DeSiTe Biological Technology Co., Ltd (DF0005, Chengdu, China). Cepharanthine, berbamine hydrochloride and tetrandrine (98\%) were from Bidepharm. MEK162 was from CSNpharm (CSN16001, Chicago, USA). Dulbecco's modified Eagle medium (DMEM) and phosphate buffer solution (PBS) were from Hyclone. Fetal bovine serum (FBS, 42F6590K), RPMI-1640 medium HEPES (1640H, 22400089) and Ham's F12K (Kaighn's) medium (F12K, 21127022) were from Gibco (Grand Island, NY, USA). Multicolour protein markers were from Absin Bioscience, Inc. (abs923, Shanghai, China) and Yeasen (20352ES76, Shanghai, China).

\section{Cell culture}

CRMM1, CRMM2 and CM2005.1 were generously provided by Professor Martine J. Jager (Leiden University Medical Center, Leiden, The Netherlands) ${ }^{47}$ and CMAS16 was provided by Professor Renbin Jia (Shanghai Ninth People's Hospital, Shanghai, China). A375 and HEK293T were obtained from American Type Culture Collection. CM-AS16 and CM2005.1 were maintained in 1640H medium, CRMM1 and CRMM2 were maintained in F12K medium, A375 and HEK293T were maintained in DMEM medium, and MRC5 was maintained in MEM medium. All mediums were supplemented with 10\% FBS. Cells were cultured at $37{ }^{\circ} \mathrm{C}$ in an incubator, in a humidified atmosphere with $5 \% \mathrm{CO}_{2}$, periodically checked for mycoplasma-free and validated by short tandem repeats 
(STR) DNA analysis at BGI Tech Solutions (Beijing Liuhe) Co., Limited.

\section{Cell viability assay}

Cells $\left(1 \times 10^{4}\right.$ cells/well $)$ were seeded in 96-well plates (100 $\mu \mathrm{L} /$ well). In succession, compounds in $100 \mu \mathrm{L}$ fresh medium were added and incubated for $72 \mathrm{~h}$. After removal of the cell culture medium, a $10 \%$ CCK- 8 (Apexbio, K1018, Houston, USA) solution in medium was administered and re-incubated for $2 \mathrm{~h}$. The absorbance at $450 \mathrm{~nm}$ was measured in a microplate reader (Biotek, Vermont, USA).

\section{Transcriptome analysis by RNA-sequencing}

CM-AS16 cells were treated with or without $8 \mu \mathrm{M}$ fangchinoline. The transcriptome analysis by RNAsequencing was performed according to a previously published method $^{48}$. The technique we used was the second-generation sequencing technology. Total RNAs were extracted using Trizol Reagent (R0016, Beyotime, Shanghai, China). Further assay and analysis were assisted by Majorbio Bio-Pharm Technology Co. Ltd (Shanghai, China) and are shown in Supplementary Dataset S1.

\section{ABPP assay}

CM-AS16 cells were lysed, incubated with probes and radiated with $\mathrm{UV}$. Then, the mixtures were combined with $300 \mu \mathrm{M}$ biotin-azide, $100 \mu \mathrm{M}$ TBTA, $1 \mathrm{mM}$ TCEP and $1 \mathrm{mM} \mathrm{CuSO}_{4}$. After that, the proteome was performed as the protocol of Capturem Streptavidin Miniprep Columns (635733, Takara, Beijing, China), separated by SDS-polyacrylamide gel electrophoresis and visualized by Coomassie blue staining. The protein bands in the whole gel were excised, followed by in-gel digestion and analysis by liquid chromatography-tandem mass spectrometry at Majorbio Bio-Pharm Technology Co. Ltd (Shanghai, China) and are shown in Supplementary Dataset S2.

\section{Cell transfection}

Taitool Bioscience (Shanghai, China) assisted in preparing FUBP2 short hairpin RNA adenovirus.

The target sequences of FUBP2 are designed as follows: shFUBP2-1, sense: 5'-CAAGATGATGCTGGATGA CAT-3';

shFUBP2-2, sense: 5'-GATGATCAAGAAGATCCA GAA-3';

shFUBP2-3, sense: 5'-GATCATCAACGACCTCCTC CA-3';

shControl, sense: 5'-AAATGTACTGCGCGTGGAG AC- $3^{\prime}$.

Cell infection was performed by direct addition of adenovirus particles to pelleted cells for $24 \mathrm{~h}$ and transduced cells were collected $24 \mathrm{~h}$ post infection for the qRT-PCR and western blot assay.

HEK293T cells were transfected with plasmids (EXZ9878-M93, GeneCopoeia, Inc., Rockville, MD, USA) with Lipofectamine 3000 (L3000015, Invitrogen, Carlsbad, CA, USA). The transfection duration was $72 \mathrm{~h}$.

\section{Antibodies}

Primary antibodies were against FUBP2 (ab150393, Abcam), c-Myc (ab32072, Abcam), RAD51 (ab133534, Abcam), BRAC1 (YT0519, ImmunoWay), GAPDH (60004-1-lg, Proteintech) and Vinculin (ab129002, Abcam).

Secondary antibodies were Cy3-labelled goat anti-rabbit IgG $(\mathrm{H}+\mathrm{L})$ (A-11035, Invitrogen), Alexa 488-conjugated anti-rabbit IgG (GB25303, Servicebio), HRP-linked Antirabbit IgG Antibody (7074 S, Cell Signaling Technology) and HRP-linked Anti-mouse IgG Antibody (7076 S, Cell Signaling Technology).

\section{MST assay}

An MST assay was conducted to test the binding affinity of fangchinoline and FUBP2 by using Monolith NT.115 (NanoTemper Technologies). FUBP2 labelled with enhanced green fluorescent protein was obtained from HEK293T cells transfected with plasmids and was used at a concentration of $10 \mu \mathrm{M}$. Fangchinoline was titrated in $1: 1$ dilutions beginning at a concentration of $500 \mu \mathrm{M}$ in $5 \%(\mathrm{v} / \mathrm{v})$ dimethyl sulfoxide (DMSO). Samples were diluted in MST assay buffer containing $25 \mathrm{mM}$ HEPES ( $\mathrm{pH}$ 7.0/7.5) supplemented with 5\% (v/v) DMSO. For the measurement, the samples were filled into premium coated capillaries.

\section{Immunofluorescence staining}

Cells were pre-treated with cisplatin for $24 \mathrm{~h}$. After that, cisplatin was removed and cells were treated with fangchinoline for $24 \mathrm{~h}$. Cells were fixed with $4 \%$ paraformaldehyde (PFA) and blocked with $5 \%$ bovine serum albumin containing $0.3 \%$ Triton X-100, then incubated with primary and secondary antibody. Hoechst 33258 (KGA211-10, KeyGEN BioTECH Co., Ltd, Nanjing, China) staining was performed before observation. Confocal fluorescence images were measured by a Leica confocal microscope (Leica TCS SPS CFSMP, Germany).

\section{Western blotting and qRT-PCR analysis}

Cells were treated with fangchinoline for $24 \mathrm{~h}$. The western blotting and qRT-PCR analysis protocols were used as described previously ${ }^{49}$. The sequences of primers are listed in Supplementary Information.

\section{Tissue immunofluorescence and Tunel assays}

The tissue immunofluorescence ${ }^{50}$ and Tunel assay ${ }^{51}$ protocols were used as described previously. 


\section{Mouse tumour xenograft studies}

Animal experiments were carried out according to the National Institutes of Health guidelines and the Association for Research in Vision and Ophthalmology guidelines.

NCG mouse tumour xenograft experiments were conducted with the approval of the Institutional Animal Care and Use Committee (IACUC) of GemPharmatech Co. Ltd (Nanjing, Jiangsu, China) (Permit Number: SYXK (Jiangsu) 2018-0027; Animal Protocol No: GPTAP016). CM-AS16 cells $\left(1.0 \times 10^{7}\right.$ in $100 \mu \mathrm{L}$ of PBS) were injected subcutaneously into male NCG mice (6-7 weeks old). When the tumours reached a volume of $\sim 80 \mathrm{~mm}^{3}$, the mice were randomized into treatment and control groups (seven mice per group). Mice in treatment groups received MEK162 $(50 \mathrm{mg} / \mathrm{kg}$, intragastric) or fangchinoline $(25 \mathrm{mg} / \mathrm{kg}$ and $50 \mathrm{mg} / \mathrm{kg}$, i.p.) once a day for 22 days. Three mice in the $25 \mathrm{mg} / \mathrm{kg}$ fangchinoline treatment group died before the end point, which might be due to the metastasis in the thymus, or weight loss or tumour enlargement.

$\mathrm{Nu} /$ nu nude mouse tumour xenograft experiments were reviewed and approved by the IACUC of the Shanghai Ninth People's Hospital Central Laboratory (Permit Number: SYXK (Shanghai) 2016-0016). A375 cells (3.0 × $10^{6}$ in $100 \mu \mathrm{L}$ of PBS) were subcutaneously inoculated into male nu/nu nude mice (5-6 weeks old) (Shanghai Jiesijie Laboratory Animal Co., Ltd). When the tumours reached a volume of $\sim 160 \mathrm{~mm}^{3}$, the mice were randomized into treatment and control groups $(n=6$ per group). Mice in treatment groups received fangchinoline $(10 \mathrm{mg} / \mathrm{kg}$ and $50 \mathrm{mg} / \mathrm{kg}$, i.p.), cisplatin $(2 \mathrm{mg} / \mathrm{kg}$, i.p.) or both once a day for 14 days.

The tumour size and body weight were monitored every 3 or 4 days. Tumour volume was calculated as $0.5 \times$ length $\times$ width ${ }^{2}$. Then, the animals were killed by $\mathrm{CO}_{2}$ and the tumour xenografts were removed and photographed. Half of each tumour tissue was used for western blotting and the other half was fixed with 4\% PFA for immunofluorescence staining. The blood samples were collected from submandibular venous plexus in NCG mice after being anesthesized with $\mathrm{CO}_{2}$.

\section{Statistical analysis}

All data are represented as mean \pm SD. Statistical analysis was conducted by Graphpad Prism 7.04 and significant differences within treatments were determined by two-way analysis of variance or Student's $t$-test. $P \leq 0.05$ was considered statistically significant.

\section{Acknowledgements}

We thank the Shanghai National Protein Science Center for the instrumental support and technical support in MST assay of this study.

\begin{abstract}
Author details
${ }^{1}$ State Key Laboratory of Bioreactor Engineering, Shanghai Key Laboratory of New Drug Design, East China University of Science and Technology, 130 Mei Long Road, Shanghai 200237, China. 'Department of Ophthalmology, Ninth People's Hospital, Shanghai Jiao Tong University School of Medicine, Shanghai 200001, China. ${ }^{3}$ College of Pharmacy and Chemistry, Dali University, 5 Xue Ren Road, Dali, Yunnan 671000, China. ${ }^{4}$ Frontiers Science Center for Materiobiology and Dynamic Chemistry, East China University of Science and Technology, 130 Mei Long Road, Shanghai 200237, China
\end{abstract}

\section{Author contributions \\ K.B. designed, performed experiments, analysed data and wrote the manuscript. Y.L., J.W., J.Y., J.S., B.L. and J.Z. performed experiments. R.L. designed, synthesized and characterized the probes. J.L., R.J. and F.M. designed, guided research and wrote the manuscript. All authors read and approved the final manuscript.}

\section{Funding}

This work was supported by grants from the National Natural Science Foundation of China $(81872747,21672064)$, the Innovative Research Team of High-level Local Universities in Shanghai, the National Special Fund for State Key Laboratory of Bioreactor Engineering (2060204), the National Natural Science Foundation of China (82003603) and the National Mega-project for Innovative Drugs of China (2019ZX09721001-004-003).

\section{Data availability}

The accession number for RNA-seq reported in this paper is GEO: GSE164340.

\section{Ethics statement}

There are no issues with human subjects in this manuscript. NCG mouse tumour xenograft experiments were conducted with the approval of the IACUC of GemPharmatech Co. Ltd (Nanjing, Jiangsu, China) (Permit Number: SYXK (Jiangsu) 2018-0027; Animal Protocol No: GPTAP016). Nu/nu nude mouse tumour xenograft experiments were reviewed and approved by the IACUC of the Shanghai Ninth People's Hospital Central Laboratory (Permit Number: SYXK (Shanghai) 2016-0016). Animal procedures were carried out according to the National Institutes of Health $(\mathrm{NIH})$ guidelines.

\section{Conflict of interest}

The authors declare no competing interest.

\section{Publisher's note}

Springer Nature remains neutral with regard to jurisdictional claims in published maps and institutional affiliations.

Supplementary information The online version contains supplementary material available at https://doi.org/10.1038/s41419-021-03653-4.

Received: 26 May 2020 Revised: 13 March 2021 Accepted: 18 March 2021 Published online: 07 April 2021

References

1. McCartney, A. C. Pathology of ocular melanomas. Br. Med. Bull. 51, 678-693 (1995).

2. Scotto, J., Fraumeni, J. F. \& Lee, J. A. Melanomas of the eye and other noncutaneous sites: epidemiologic aspects. J. Natl Cancer Inst. 56, 489-491 (1976).

3. Vora, G. K., Demirci, H., Marr, B. \& Mruthyunjaya, P. Advances in the management of conjunctival melanoma. Sun. Ophthalmol. 62, 26-42 (2017).

4. Hu, D. N., Yu, G., McCormick, S. A. \& Finger, P. T. Population-based incidence of conjunctival melanoma in various races and ethnic groups and comparison with other melanomas. Am. J. Ophthalmol. 145, 418-423 (2008).

5. Tolleson, W. H. Human melanocyte biology, toxicology, and pathology. J. Environ. Sci. Health C. Environ. Carcinog. Ecotoxicol. Rev. 23, 105-161 (2005).

6. Nair, B. C. J. et al. Conjunctival melanoma: bladder and upper urinary tract metastases. J. Clin. Oncol. 29, e216-e219 (2011).

7. Shields, J. A., Shields, C. L., \& De Potter, P. Surgical management of conjunctival tumors. Arch. Ophthalmol. 115, 808-815 (1997). 
8. Burattini, S., Battistelli, M. \& Falcieri, E. Morpho-functional features of in-vitro cell death induced by physical agents. Curr. Pharm. Des. 16, 1376-1386 (2010).

9. Fernandes, B. F. et al. Local chemotherapeutic agents for the treatment of ocular malignancies. Sun. Ophthalmol. 59, 97-114 (2014)

10. Brouwer, N. J. et al. Treatment of conjunctival melanoma in a Dutch referral centre. Br. J. Ophthalmol. 102, 1277-1282 (2018).

11. Zhou, C. D., Wang, Y. X., Jia, R. B. \& Fan, X. Q. Conjunctival melanoma in Chinese patients: local recurrence, metastasis, mortality, and comparisons with Caucasian patients. Invest. Ophthalmol. Vis. Sci. 58, 5452-5459 (2017).

12. Sarkisian, S. \& Davar, D. MEK inhibitors for the treatment of NRAS mutant melanoma. Drug Des. Devel. Ther. 12, 2553-2565 (2018).

13. Lake, S. L. et al. Multiplex ligation-dependent probe amplification of conjunctival melanoma reveals common BRAF V600E gene mutation and gene copy number changes. Invest. Ophthalmol. Vis. Sci. 52, 5598-5604 (2011).

14. Griewank, K. G. et al. Conjunctival melanomas harbor BRAF and NRAS mutations and copy number changes similar to cutaneous and mucosal melanomas. Clin. Cancer Res. 19, 3143-3152 (2013).

15. Jakob, J. A. et al. NRAS mutation status is an independent prognostic factor in metastatic melanoma. Cancer 118, 4014-4023 (2012).

16. Thomas, N. E. et al. Association between NRAS and BRAF mutational status and melanoma-specific survival among patients with higher-risk primary melanoma. JAMA Oncol. 1, 359-368 (2015).

17. Queirolo, P. \& Spagnolo, F. Binimetinib for the treatment of NRAS-mutant melanoma. Expert Rev. Anticancer Ther. 17, 985-990 (2017).

18. Maleka, A., Åström, G., Byström, P. \& Ullenhag, G. J. A case report of a patient with metastatic ocular melanoma who experienced a response to treatment with the BRAF inhibitor vemurafenib. BMC Cancer 16, 634-638 (2016).

19. Mor, J. M. \& Heindl, L. M. Systemic BRAF/MEK inhibitors as a potential treatment option in metastatic conjunctival melanoma. Ocul. Oncol. Pathol. 3 133-141 (2017)

20. Li, Y. et al. Characterization of a conjunctival melanoma cell line CM-AS16, newly-established from a metastatic Han Chinese patient. Exp. Eye Res. 173, 51-63 (2018)

21. Chen, F. et al. Small-molecule targeting of a diapophytoene desaturase inhibits S. aureus virulence. Nat. Chem. Biol. 12, 174-179 (2016).

22. Wang, $\mathrm{Y}$. et al. Discovery of potent Benzofuran-derived diapophytoene desaturase $(\mathrm{CrtN})$ inhibitors with enhanced oral bioavailability for the treatment of methicillin-resistant Staphylococcus aureus (MRSA) infections. J. Med. Chem. 59, 3215-3230 (2016).

23. Mérarchi, M. et al. Molecular targets modulated by fangchinoline in tumor cells and preclinical models. Molecules 23, 2538 (2018).

24. Avigan, M. I., Strober, B. \& Levens, D. A far upstream element stimulates c-myc expression in undifferentiated leukemia cells. J. Biol. Chem. 265, 18538-18545 (1990).

25. Luoto, K. R. et al. Tumor cell kill by c-MYC depletion: role of MYC-regulated genes that control DNA double-strand break repair. Cancer Res. 70, 8748-8759 (2010).

26. Nareyeck, G., Wuestemeyer, H., von der Haar, D. \& Anastassiou, G. Establishment of two cell lines derived from conjunctival melanomas. Exp. Eye Res. 81, 361-362 (2005)

27. Keijser, S., Maat, W., Missotten, G. S. \& de Keizer, R. J. A new cell line from a recurrent conjunctival melanoma. Br. J. Ophthalmol. 91, 1566-1567 (2007)

28. Hosoya, N. \& Miyagawa, K. Targeting DNA damage response in cancer therapy. Cancer Sci. 105, 370-388 (2014).

29. Bhattacharyya, A., Ear, U. S., Koller, B. H., Weichselbaum, R. R. \& Bishop, D. K. The breast cancer susceptibility gene BRCA1 is required for subnuclear assembly of
Rad51 and survival following treatment with the DNA cross-linking agent cisplatin. J. Biol. Chem. 275, 23899-23903 (2000).

30. Cravatt, B. F., Wright, A. T. \& Kozarich, J. W. Activity-based protein profiling: from enzyme chemistry to proteomicchemistry. Annu. Rev. Biochem. 77, 383-414 (2008).

31. He, L. et al. Loss of FBP function arrests cellular proliferation and extinguishes c-myc expression. EMBO J. 19, 1034-1044 (2000).

32. Gabay, M., Li, Y. \& Felsher, D. W. MYC activation is a hallmark of cancer initiation and maintenance. Cold Spring Harb. Perspect. Med. 4, a014241 (2014).

33. Carey, J. P. W. et al. Synthetic lethality of PARP inhibitors in combination with MYC blockade is independent of BRCA status in triple-negative breast cancer. Cancer Res. 78, 742-757 (2018).

34. Li, M., Li, A., Zhou, S., LV, H. \& Yang, W. SPAG5 upregulation contributes to enhanced c-MYC transcriptional activity via interaction with c-MYC binding protein in triple-negative breast cancer. J. Hematol. Oncol. 12, 14 (2019).

35. Niedernhofer, L. J., Lalai, A. S. \& Hoeijmakers, J. H. J. Fanconi anemia (cross) linked to DNA repair. Cell 123, 1191-1198 (2005).

36. Duxin, J. P. \& Walter, J. C. What is the DNA repair defect underlying Fanconi anemia? Curr. Opin. Cell Biol. 37, 49-60 (2015).

37. Lohse, I. et al. BRCA1 and BRCA2 mutations sensitize to chemotherapy in patient-derived pancreatic cancer xenografts. Br. J. Cancer 113, 425-432 (2015).

38. Moynahan, M. E., Chiu, J. W., Koller, B. H. \& Jasin, M. Brca1 controls homologydirected DNA repair. Mol. Cell 4, 511-518 (1999).

39. Ledermann, J. A., Drew, Y. \& Kristeleit, R. S. Homologous recombination deficiency and ovarian cancer. Eur. J. Cancer 60, 49-58 (2016).

40. Takenaka, T. et al. Combined evaluation of Rad51 and ERCC1 expressions for sensitivity to platinum agents in non-small cell lung cancer. Int. J. Cancer 121, 895-900 (2007).

41. Xiao, M. et al. Let-7e sensitizes epithelial ovarian cancer to cisplatin through repressing DNA double strand break repair. J. Ovarian Res. 10, 24 (2017).

42. Wang, B. et al. Artesunate sensitizes ovarian cancer cells to cisplatin by downregulating RAD51. Cancer Biol. Ther. 16, 1548-1556 (2015).

43. Liu, W. et al. KSRP modulates melanoma growth and efficacy of vemurafenib. Biochim. Biophys. Acta Gene Regul. Mech. 1862, 759-770 (2019).

44. Olaussen, K. A. et al. DNA repair by ERCC1 in non-small-cell lung cancer and cisplatin-based adjuvant chemotherapy. N. Engl. J. Med. 355, 983-991 (2006).

45. Bryant, H. E. et al. Specifc killing of BRCA2-defcient tumours with inhibitors of poly(ADP-ribose) polymerase. Nature 434, 913-917 (2005).

46. Dupré, A. et al. A forward chemical genetic screen reveals an inhibitor of the Mre11-Rad50-Nbs1 complex. Nat. Chem. Biol. 4, 119-125 (2008).

47. Cao, J. et al. Targeting of the MAPK and AKT pathways in conjunctival melanoma shows potential synergy. Oncotarget 8, 58021-58036 (2017).

48. Hao, S. et al. Transcriptome analysis of phycocyanin-mediated inhibitory functions on non-small cell lung cancer A549 cell growth. Mar. Drugs 16, 511 (2018).

49. Liu, W. et al. Verapamil extends lifespan in Caenorhabditis elegans by inhibiting calcineurin activity and promoting autophagy. Aging (Albany NY) 12 5300-5317 (2020).

50. Ma, Y. et al. Extracellular vesicles from human umbilical cord mesenchymal stem cells improve nerve regeneration after sciatic nerve transection in rats. $J$. Cell. Mol. Med. 23, 2822-2835 (2019).

51. Yang, Q. et al. Effects of the different-sized external stents on vein graft intimal hyperplasia and inflammation. Ann. Transl. Med. 8, 102 (2020). 